\title{
La caverne de Maître Jehan ou l'étrange ballet des mots du Mirouer de la mort (1519-1575)
}

Maitre Jehan's cave and the strange dance of the poem Mirouer de la mort (1519-1575)

Yves Le Berre

\section{OpenEdition}

\section{Journals}

Édition électronique

URL : http://journals.openedition.org/lbl/408

DOI : $10.4000 / \mathrm{lbl} .408$

ISSN : 2727-9383

\section{Éditeur}

Université de Bretagne Occidentale - UBO

\section{Édition imprimée}

Date de publication : 1 octobre 2018

Pagination : 101-114

ISBN : 979-10-92331-40-0

ISSN : 1270-2412

\section{Référence électronique}

Yves Le Berre, « La caverne de Maître Jehan ou l'étrange ballet des mots du Mirouer de la mort

(1519-1575) », La Bretagne Linguistique [En ligne], 22 | 2018, mis en ligne le 01 mai 2020, consulté le 01 octobre 2020. URL : http://journals.openedition.org//bl/408 ; DOI : https://doi.org/10.4000//bl.408

La Bretagne Linguistique est mise à disposition selon les termes de la Licence Creative Commons Attribution 4.0 International. 


\section{La caverne de Maître Jehan ou l'étrange ballet des mots du Mirouer de la mort (1519-1575)}

Le Mirouer de la mort est un poème didactique de 3602 vers groupés en sizains d'octosyllabes et de décasyllabes, ou en quatrains de dodécasyllabes, écrit en breton au début du XVI ${ }^{\mathrm{e}}$ siècle et bâti en quatre parties thématiques : la mort physique, le jugement dernier, l'enfer, le paradis. La page de titre est en français, en latin et en breton; elle est suivie d'un bois gravé dont le commentaire est en français ; tous les titres courants et les nombreux intertitres sont en français. Cela peut surprendre. Mais dans les deux manuscrits dramatiques qui sont à peu près contemporains de l'impression du Mirouer, Buez, sant [es] Nonn hac ez. map deuy [Vie de sainte Nonne et de son fils Dévy] et An Bubez sant Gwenôlé Abat quentaf eus a Lantevennec [La Vie de saint Gwénolé, premier abbé de Landévennec], les didascalies sont en latin. Ce qui me suggère deux hypothèses entre lesquelles j'hésite à choisir, et qui ne sont peutêtre pas contradictoires :

a. Il y aurait eu une version française du Mirouer imprimée à Cuburien avant la version bretonne, et dont les plombs auraient été réutilisés pour la partie bretonne ${ }^{1}$.

* Professeur émérite de breton et celtique, CRBC (EA 4451), UBO.

1. En 1623 un imprimeur publie une Vie de saint Yves écrite par Pierre de La Haye, puis, la même année, sa traduction bretonne réalisée par Tanguy Guéguen. Mais dans le cas du Mirouer, rien ne reste de l'éventuelle édition française, pas même une allusion. 
b. La lecture étant ce qu'elle était à cette époque, il se peut qu'une médiation ait été prévue entre le livre et ses destinataires. Au théâtre l'assistance d'un bon lecteur était nécessaire aux comédiens analphabètes, afin de les aider à mémoriser leur texte. Les clercs étaient ceux qui étaient le plus à même de tenir ce rôle. Dans le cas d'un poème spirituel de facture complexe, comme le Mirouer, chaque partie devait être expliquée et commentée aux publics dépourvus d'éducation scolaire. Les sous-titres, comme les didascalies au théâtre, seraient donc destinés à ce seul médiateur.

Nous avons jusqu'à présent connaissance de trois états successifs du poème :

a. L'original supposé de 1519, écrit à la plume par un « Mæstre Iehan an Archer Coz ", de Plougonven. Nous ne savons pour ainsi dire rien de l'auteur (dont le nom même n'est pas expliqué) et ne verrons sans doute jamais son manuscrit.

b. L'impression de 1575 réalisée à l'imprimerie du couvent franciscain de Cuburien, qui ne doit pas être tout à fait fidèle au manuscrit du vieux Jehan. D'une part les accents grave et aigu n'existaient pas plus en son temps que le point d'interrogation, les lettres collées ou la virgule ; d'autre part de légères altérations, relevant peut-être d'automatismes badumesques du moine typographe ${ }^{2}$, font ici et là hoqueter l'admirable mécanique des rimes entrelacées.

c. L'édition savante de 1914 signée par Émile Ernault ${ }^{3}$, qui présente une introduction de 30 pages, alignant 35 réflexions où il est beaucoup question de versification, puis une transcription de l'unique exemplaire survivant du stock de 1575, lequel, après avoir appartenu à la famille de Kerdanet, repose désormais en sûreté à la Bibliothèque nationale. En face de cette transcription, un texte français qu'il est difficile de qualifier de traduction, qui serait plutôt une transverbation : Ernault se contente le plus souvent de remplacer les mots bretons par des mots français sans en changer l'ordre, ce qui donne par exemple :

Envie et malice, depuis, sont dans le misérable odieux,

2. "Don ren de tron hac euyt hon gounit » (M3446) Don, tron et hon rimaient forcément avec gonit, dans l'original.

3. Émile ERNAULT, Le mirouer de la mort, poème breton du XVIe siècle, publié d'après l'exemplaire unique, Paris, H. Champion, 1914. 
D'être venu à une forte peine et gémissement, sans aucun ménagement... ${ }^{4}$

(M2231-32)

Enfin des notes lexicologiques de bas de page, abondantes mais pas toujours très éclairantes, complétées par 37 pages d'additions et corrections qui ne facilitent pas non plus la lecture du volume.

Le petit travail que je présente ici est initialement motivé par mon étonnement face à l'apparente incapacité d'Ernault à traduire le Mirouer dans un français syntaxiquement construit. Les autres grands textes de la même époque qu'il a publiés sont trois drames religieux : la Vie de sainte Nonne (1887), la Vie de sainte Barbe $(1887)^{5}$ et la Vie de saint Gwénolé (1832-1934) ${ }^{6}$. Sans être très travaillées, les traductions qu'il en donne sont lisibles et cohérentes. Je suis donc curieux de comprendre pourquoi ce texte, le seul poème qu'il ait édité, lui donne autant de fil à retordre.

Mon hypothèse est que cette difficulté a une cause principale, voire unique : Maitre Jehan prend sciemment plaisir à nous troubler en jouant sur la place des mots dans la chaîne écrite. Ce jeu savant, que connaissent et pratiquent nombre de poètes qui lui sont contemporains, en Bretagne et dans d'autres régions, ne peut assurément pas être réduit à la manifestation d'une maladresse due à l'incompétence, ou à un simple exercice formel relevant d'un maniérisme ou de la bella maniera dont l'Italie fera très bientôt don à la France. Ma conclusion proposera de lui donner une autre signification, socioculturelle et mystico-philosophique, plus large et plus profonde.

\section{Un mot déplacé}

Placer arbitrairement un mot devant celui qu'il suit d'ordinaire est un vieux truc stylistique qu'on trouve aussi bien chez Cicéron :

Scripsi... quodam in libello...

[J'ai écrit dans un certain traité...]

(De Oratore, XXI)

4. Il s'agit de l'état d'esprit de Satan, chassé du paradis.

5. CEuvres de jeunesse : il a alors 35 ans.

6. CEuvre ultime : il mourra quatre ans plus tard. 
que chez Salvatore Adamo, le chanteur belge de la génération yéyé :

Tu ne viendras pas ce soir

Je crie mon désespoir

Tombe la neige

Impassible manège

(Salvatore Adamo, 1992)

Il est vrai que ce dernier a pu s'inspirer du célèbre refrain d'Apollinaire :

Vienne la nuit sonne l'heure

Les jours s'en vont je demeure.

(«Sous le pont Mirabeau », Alcools, 1913)

C’est dire l'universalité, la longévité et la popularité de ce procédé que les rhétoriciens nomment byperbate.

Il est très couramment utilisé dans tous les textes de la première littérature du breton, entre le XVe et le XVII ${ }^{\mathrm{e}}$ siècle, et le Mirouer en contient un grand nombre, touchant surtout la fonction qualificative :

Endeues meur á den, lamet yen á penet ${ }^{7}$

[(la pénitence) A à de nombreux hommes évité dures de punitions]

Ernault : «a retiré plus d'un homme, froidement, de châtiment »

(M2596)

Dison he pouruisionou

[(Il faut mépriser le monde et) délicates ses nourritures]

Ernault : «Sans peine, et ses richesses »

En yffernn eternal : doen scandal poan calet,

Hep gallout caffout lem, ouz en em clem remet.

[En enfer éternels endurer les tourments d'une sévère punition,

Sans pouvoir obtenir, vivement en se plaignant, rémission]

Ernault : «Dans l'enfer éternel... certes...»

(M2585-M2586)

L'hyperbate permet souvent d'obtenir la troisième rime interne : eternal... scandal... calet. De même au vers suivant : lem... clem... remet.

7. Double hyperbate. L'ordre non poétique serait En deues lamet meur à den à penet yen. 
Un mot fréquent comme Yffern « appelle » souvent des mots avec lesquels il rime : cistern, buernn, espern, cern, bern, eternel. Mais ceux-ci ne le qualifient jamais, contrairement à yen, profont, couen, puant, avec lesquels il ne rime pas.

La difficulté est qu'il faut se soumettre à un entraînement intensif pour repérer sans hésitation le nom qui est qualifié; mais on reste étonné de voir Ernault, lecteur professionnel de prose et de poésie latines, tomber aussi souvent dans le piège :

An eneff en é bro en reo, ha so creff

[L'âme, qui est forte, l'emmènera ${ }^{8}$ dans sa patrie]

Ernault : «l'âme la conduira dans son pays, ce qui est fort »

Alors qu'en latin on sait que quodam va avec libello, grâce à la désinence $o$ de l'ablatif masculin, rien de tel en breton. Ce jeu est donc bien plus facile à pratiquer avec des langues flexionnelles.

\section{Un syntagme déplacé}

On dit toujours que la syntaxe du breton est « souple », par opposition sans doute à celle du français qui serait « raide ». Mais les règles qui encadrent cette souplesse ne rendent pas du tout compte de ce qui se passe dans le Mirouer, à des niveaux supérieurs à celui du mot. Ainsi dans la grammaire banale le déplacement d'un élément de la proposition en position topique ou thématique ne change-t-il pas l'ordre des autres éléments. Les formules les plus courantes, SVO, VSO ou OVS sont toutes des variantes marquées de SVO (en réponse à une question, par exemple). Ce qui se lit dans les exemples suivants est très différent:

Ouz an guir Doe da nep à voe leal

[(joie et béatitude sont accordées) Au vrai Dien à ceux qui furent fidèles ...]

Hac hon buhez yuez, an boll desyou

Hac an bet man noman he boll yoaou

[Et de notre vie ainsi tous les jours

Et de ce monde dorénavant tous ses plaisirs... (sont sans valeur auprès des joies célestes)]

8. Il s'agit de la chair, donc du corps. 
Entre $n$ Aelez / hep finuez, / ha bezaff ${ }^{9}$

[Parmi les anges / à tout instant / et se trouver]

Apollinaire utilise lui aussi ce procédé (une sorte d'anacoluthe) :

Les mains dans les mains restons face à face

Tandis que sous

Le pont de nos bras passe

Des éternels regards l'onde si lasse

(«Sous le pont Mirabeau », Alcools, 1913)

\section{Une proposition déplacée}

Une proposition est déplacée dans la phrase; une autre prend évidemment sa place :

An tan á vezpeinctet, ouz tan gruet en bet man,

Nedeu da veraff quet, comparaichet ledan:

[Le feu qui est peint / à un feu allumé en ce monde

Ne saurait être / comparé en rien]

(M1999-2000)

À lire dans l'ordre 1-3-4-2.

En gallout drouc Alez, nos ha dez, truezus:

So cruel ho guelet, pepret ha morchedus,

[À la merci des démons / faisant sans cesse pitié

À l'aspect repoussant / et toujours affligés ${ }^{10}$ ]

À lire dans l'ordre 1-3-2-4.

(M1616-1617)

Hac ez eux niuer bras, an queiz azgas caset,

So leun plen á venim, na guell bout estimet:

[Et il y a un nombre immense / de misérables réprouvés,

Qui sont pleins de méchanceté / incommensurable]

(M2243-2244)

À lire dans l'ordre $1-4-2-3$.

Elle peut en outre être disjointe :

9. Ces trois syntagmes sont à lire de la droite vers la gauche, comme au v. $3309:$ En

leuenez, oar lahez,pan vę̧hont 'Dans l'allégresse, là-haut, quand ils seront'.

10. Les deux hémistiches de gauche parlent des démons, les deux de droite des damnés. 


\section{En figur an traman, noman an Romanet,}

Guez á voe ne voe goap, pan croéent an Papet:

Diraze tan en stoup, á veze adoubet:

Enit bumilite, ez veze discuezet ${ }^{11}$.

[Pour symboliser cela / alors les Romains

D'autrefois, régulièrement / quand ils intronisaient les papes

Devant eux un feu d'étoupe / qui était préparé

Pour (les rappeler à) l'humilité / leur était montré]

Les 4 derniers hémistiches sont à lire dans l'ordre $1-4-2-3$.

De nombreuses combinaisons sont logiquement possibles, mais toutes ne sont pas réalisées dans le poème. Si le travail en vaut la peine, j'en ferai ultérieurement un relevé et un classement complets.

Le prédécoupage du texte en vers et en hémistiches rend le montagedémontage plus facile à réaliser dans les vers longs. Le Mirouer contient des dizaines, peut-être des centaines de ces figures qui concernent généralement quatre hémistiches consécutifs, parfois davantage.

\section{La latéralisation}

Les formules $1-3-2-4$ et $1-3-4-2$ sont de loin les plus fréquentes dans l'ensemble du poème. La répétition d'une lecture latéralisée par colonne (les 2 hémistiches de gauche, puis les 2 hémistiches de droite) crée une certaine habitude qui la rend plus facile à repérer. Voici deux autres exemples de formules latéralisées :

Allas ba ne tal quet, quement so en bet man,

Vn Eneff en effet, á ve net miret glan :

Rac se he euezhat, groa en mat ouz Sathan,

Hac en dyuez ezy, dan ty maz eux diboan.

[Or donc, une âme qu'on garde bien pure

Ne vaut-elle pas, en vérité, tous les biens de ce monde?

Fais donc tout pour bien la protéger de Satan,

Et quand tout sera fini tu partiras pour la demeure où règne la félicité.]

À lire dans l'ordre $1-3-4-2$.

\footnotetext{
11. La présence de eq au début du dernier hémistiche montre que l'ordre «normal»
} pour l'auteur, avant déplacement, était : diraze ez veze discuezet tan en stoup... 
Entre an holl poanyou, han tourmantou couen,

Diouz compas las ha scuezr, a punis pechezrien:

Hac ho groa da bout stanc, bep confranc gant ancquen ${ }^{12}$

Muyguet poan nep heny, bac aray bizuicquen.

[Parmi toutes les peines et les tortures affreuses

Qui punissent rudement ${ }^{13}$ au compas et à l'équerre les pécheurs

Et qui les accablent / d'une douleur inapaisable

Plus qu'aucune autre / qui durera à jamais...]

(M1175-1178)

À lire dans l'ordre $5-7-6-8$.

Ce qui autorise l'auteur à se payer d'audace en l'étendant, non plus sur quatre, mais sur six hémistiches :

Compsou Iob dan propos, so dymp clos exposet

A donge nede gaou, è boll euffrou gnouet:

Dre'n pez naoa certen, pan vise tremenet,

Ac acceptabl visent, da roe'n sent ententet.

Job nous parle très clairement de ce sujet :

Il était réellement inquiet / toutes ses œuvres, une fois exposées

Parce qu'il n'était pas certain / après son trépas,

Qu'elles seraient agréables / au roi des saints, voyez-vous.]

(M1335-1338)

À lire dans l'ordre 3-5-7-4-6-8 ou 3-5-7-8-6-4.

Et même sur huit hémistiches :

An corff á diaues : gant angoes han esgoar,

A vezo cog brochet, á se quet na gret mar :

Gant seziou dagou lem : hep esquem dimemoar,

Naoun pep lech : ha sechet en bet ne caffet par.

[Le corps, du dehors / causant souffrances et douleurs

Sera cruellement taraudé / vous pouvez me croire

Par des traits et des poignards effilés / incessantes, inimaginables

Une faim et une soif constantes / dont on ne trouverait pas

d'équivalents en ce monde.]

(M2367-2370)

À lire dans l'ordre $1-3-5-7-2-4-6-8$.

Yuez he pridiry, heruez maz studiaff,

Ara meur á heny, hep muy contrediaff :

12. Ici, en outre, une hyperbate.

13. Hyperbate: las qualifie punis. 


\section{Da ober pinigenn, en glenn quent gourfennaff,}

A pep tu é bubez, yuez na finuezaff.

[En outre, le fait d'y penser ${ }^{14} /$ je le constate

Incite beaucoup de gens / sans doute aucun

À faire pénitence / ici-bas avant de trépasser

Étant encore en vie / donc avant de mourir]

$$
\text { À lire dans l'ordre } 1-3-5-7-2-4-6-8 \text {. }
$$

\section{Le brouillage syntaxique}

Virgile, que maître Jean nomme an mastr á poetry [le maître de la poésie] (M142) commence l'Énéide par ces mots :

Arma virumque cano, Troiae qui primus ab oris

Italiam, fato profugus, Laviniaque venit

litora...

Ce qui, traduit littéralement, donne :

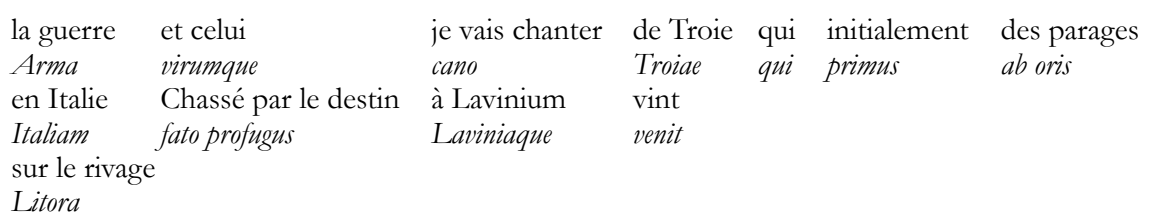

Et, une fois remis en ordre :

"Je vais chanter la guerre et celui qui, chassé par le destin, vint initialement des parages de Troie en Italie, à Lavinium, sur le rivage...»

Deux mille ans plus tard, Raymond Queneau a utilisé dans ses Exercices de style ${ }^{15}$ un procédé tout proche, qu'on nomme browillage syntaxique ou synchyse ('confusion', en grec):

« Ridicule jeune homme, que je me trouvai un jour sur un autobus de la ligne $\mathrm{S}$ bondé par traction peut-être cou allongé, au chapeau la cordelière, je remarquai un. Arrogant et larmoyant d'un ton, qui se trouve à côté de lui, contre ce monsieur, proteste-t-il. Car il le pousserait, fois chaque que des gens il descend. Libre il s'assoit et se précipite vers une place, ceci dit. Rome (Cour de) je le rencontre plus tard deux heures à son pardessus un bouton d'ajouter un ami lui conseille. »

14. Aux souffrances des damnés.

15. Raymond QuenEAU, Exercices de style, Paris, Gallimard, 1947, p. 16. 
Il ne l'a pas inventé, car l'auteur du Mirouer adore déjà délivrer ses phrases dans un désordre délibéré qui oblige l'auditeur à remettre les pièces du puzzle à leur place, pour faire apparaitre le sens :

Er bezcoaz calon be contaff, Car jamais cœur la décrire

Na lagat guelet à credaff :

Ni œil contempler, j'en suis sûr,

Ne gallas scaff bihanaff yoa.

Na scouarnn mar dreu è cleuet,

So gant Doe da den ordrenet:

Mar car è caret en bet ma. À condition qu'il veuille bien l'aimer en ce monde.

Ne put la plus infime joie, en vérité,

$\mathrm{Ni}$ si fine oreille la percevoir,

Qui a été préparée par Dieu pour l'homme,

(M2763-68)

Hyperbate dans un ensemble latéralisé, qui donne une lecture verticale sur trois colonnes :

Hac en treit ereou, da douen poanyou couen:

Hac en daou dornn fournis : pez languis da cristen?

[Et aux pieds / des liens / pour infliger d'horribles douleurs

Et aux mains / solides / quelle souffrance pour l'homme !]

(M2413-2414)

Distribution aléatoire des qualificatifs :

Affliction en nep façon confus,

Na bout nepret dre nep fat morchedus:

Na caffaous poanius dre nep vsaig.

Ne guell nepret gouzuezet naret mar,

Dre maz ynt yach ober deze glachar:

Er roe nouar en bo gloar diparaig ${ }^{16}$.

Nulle affliction quelque peu fâcheuse

$\mathrm{Ni}$ être pour quelque raison accablante Ni pénible, douloureuse en aucune façon Ne peut jamais assurément, sachez-le Leur causer de chagrin, car ils sont forts ; C'est que le souverain de la terre est leur [incomparable puissance. (M3195-3200)

Doe aternal leal hep contraly, Dieu éternelle, parfaite, légitime, A gloar certen aroy da pep heny: D'allégresse accordera sûrement à chacun d'eux Infinit muy eguet na studiont ${ }^{17}$. Infinie, plus qu'il n'en désire

Éloignement du verbe-nom et de son auxiliaire :

16. On peut facilement « remettre en ordre » les éléments principaux de la phrase : $N e$ guell affliction confus ober deze glachar, na bout morchedus na caffaous, poanius.

17. L'ordre non poétique serait : Doe a roy a gloar da pep heny muy eguet na studiont [Dieu accordera à chacun d'eux davantage d'allégresse qu'il n'en désire]. Et le brouillage est redoublé par la distribution volontairement aberrante des cinq qualificatifs de gloar: aternal, leal, hep contraly, certen, infinit. 
An Boet man glan dre contananc, Loedaff na sechaff diauanc: Eyut nep abundanc lancet. Bizhuicquen ne ra à tra sur,
Cette nourriture, pure par nature, Moisir ni se dessécher désavantageusement, Quelque profus que soit son jaillissement, Jamais ne fait, assurément ;

(M3046-3048).

Ancouffnez oll oar vn stroll bo holl po[an] Oublier totalement, d'un seul coup,

Hodeues quet gouraffet en bet man: Qu'ils ont endurées ici-bas, [toutes leurs peines Araint breman noman han bihanez. Ils feront instantanément, et les misères.

On comprend pourquoi il est possible d'y voir véritable puzzle à reconstituer. Et Molière, un siècle et demi après notre poète de Plougonven et trois siècles avant Queneau, nous donne un échantillon des jeux de syntaxe grâce auxquels on recherchait toujours la distinction au sein de la foule des courtisans du Roi-Soleil, et en outre l'attention prometteuse de leurs épouses et de leurs filles :

«Le maitre de philosophie :

On les peut mettre premièrement comme vous avez dit : Belle Marquise, vos beaux yeux me font mourir d'amour. Ou bien : D'amour mourir me font, belle Marquise, vos beaux yeux. Ou bien : Vos yeux beaux d'amour me font, belle Marquise, mourir. Ou bien : Mourir vos beaux yeux, belle Marquise, d'amour me font. Ou bien : Me font vos yeux beaux mourir, belle Marquise, d'amour ${ }^{18}$.»

On admirera tout de même rétrospectivement les lecteurs du vieux Jehan qui parvenaient à suivre son discours. À moins que dès le départ le poème n'ait été conçu comme un exercice de logique destiné à être décrypté dans l'intimité.

\section{Conclusion}

On lit souvent des phrases plutôt allusives concernant le néoplatonisme des lettrés de la fin du XVe siècle et du début du XVI ${ }^{\mathrm{e}}$. Je crois que nous disposons avec le Mirouer breton d'un exemple concret de création poétique illustrant une vision philosophique de la réalité 
du monde clairement inspirée par les principes d'immanence et de transcendance, illustrés chez Platon, entre autres passages, par le célèbre «mythe de la caverne ${ }^{19} »$.

Peu importe finalement que Jehan ait emprunté le propos et le plan de son exposé à Denis de Leeuvis ou, ce que je crois, à l'un de ses épigones francophones. Ce qui compte, pour nous qui le lisons aujourd'hui, c'est le travail de pédagogie mystique qu'il a réalisé, sous le propos plutôt banal qui lui sert de prétexte, dans une langue qui n'avait encore jamais été soumise à de telles exigences et qui ne le sera plus jamais.

Ce travail se manifeste dans la très grande tension qui oppose deux des dimensions formelles du poème. D'une part son chaos syntaxique méthodiquement organisé, ainsi que nous venons de le voir ; d'autre part son corsetage métrique extrêmement rigoureux. On aurait pu s'attendre à ce que Bubez. Mabden, qui traite du même sujet avec les mêmes moyens linguistiques et prosodiques, présente la même tension, mais ce n'est pas le cas : si sa versification est aussi rigoureuse que celle du Mirouer, sa syntaxe n'est troublée ici et là que par les nécessités de bâtir des strophes régulières tout en produisant un discours compréhensible, pas de façon systématique.

J'ai déjà eu l'occasion ${ }^{20}$ de dire ce que je pense de cette technique de versification : elle ambitionne d'extraire la langue de sa trivialité quotidienne, d'en faire un instrument de transport émotionnel vers la sphère du sacré, donc de donner au lecteur ou à l'auditeur un avant-goût des splendeurs du paradis et des horreurs de l'enfer, afin de l'encourager à emprunter les voies du repentir et de la pénitence. Ma thèse est que cet ordre puissant, où tout est à sa place, est l'allégorie de la toute-puissance divine, source et garante de l'ordre général de la Création ; il vise par conséquent à rendre sensible ce qu'on nomme la transcendance. Le système des rimes et des rythmes du Mirouer est d'évidence trop bien

19. Platon, Euvres complètes, Tome I, traduction nouvelle et notes par Léon Robin, Bibliothèque de la Pléiade, Paris, NRF, 1953, «La République », livre VII, p. 1101 et suivantes.

20. Dans Profanation et sacralisation. Réflexion sur une traduction bretonne du Stabat Mater. Essai de sociolinguistique historique expérimentale, Brest, CRBC, 2009 et dans La Passion et la Résurrection bretonnes de 1530, suivies de trois poèmes, Brest, CRBC, 2011. 
imité des rouages créés dans l'univers par le Grand Horloger ${ }^{21}$ pour qu'on puisse n'y voir que le loisir insignifiant d'un moine accablé par l'ennui de la vie conventuelle.

Ce qui est nouveau dans le Mirouer, et qui restera unique, c'est que cette impeccable régularité est sans cesse confrontée à l'allégorie de l'imperfection du monde tel que les hommes le gèrent, allégorie qui s'objective dans le malaise qu'on ressent en réalisant que dans ce discours des centaines d'éléments ne sont pas à leur place. Nous sommes ici dans le monde du désordre, de l'illusion, du faux-sens, de la difficulté à interpréter le cours des choses, donc de l'immanence humaine. Au lieu de ne lire que de gauche à droite et de haut en bas, on lit aussi de droite à gauche ${ }^{22}$ et de bas en haut, parfois en diagonale; au lieu de suivre les lignes on parcourt des colonnes; on ne sait plus qui qualifie quoi ; les membres des phrases sont jetés comme au hasard dans les strophes...

Tel le prisonnier libéré, se dirigeant vers l'entrée de la caverne, habituant peu à peu ses yeux à l'éblouissante lumière divine, et prenant progressivement conscience de l'inanité de tout ce qu'il croyait savoir et comprendre du monde réel, le lecteur du Mirouer interprète le chaos des mots, les remet patiemment à leur place et décrypte les indices que la transcendance a semés dans l'immanence pour permettre à son entendement de se hisser, moyennant de sérieux et constants efforts, vers la Vérité. Cet entrelacement de l'ordre et du désordre se manifeste superbement dans d'autres arts que la poésie, au moment où le gothique flamboyant commence à annoncer le baroque: en architecture, en musique et en peinture. Un tel travail n'est pas à la portée du premier venu.

De même est-il trop complexe et trop subtil pour être interprété par l'auditeur au vol d'une récitation orale. Il suppose donc, au moins dans un premier temps, un mode de lecture lent et solitaire - mais pas forcément silencieux - réservé à un happy few très cultivé, lecteur averti

21. L'expression n'apparaît en vérité que dans les Méditations métaphysiques de Descartes (1641), mais il y a alors bien longtemps déjà qu'on se représente l'univers comme une grande mécanique astrale (voir par exemple M2183-2186).

22. Léonard de Vinci, mort à Amboise justement en 1519, écrivait ses notes quotidiennes en toscan et en écriture spéculaire, c'est-à-dire de droite à gauche, en inversant les lettres. Ce qui était n'était pas très difficile pour un Florentin gaucher et rendait son texte quasiment indéchiffrable (sauf au moyen d'un miroir). 
des rhétoriqueurs contemporains du poète de Plougonven et habile au jeu de la reconstruction du sens rigoureux plus ou moins bien caché sous des apparences désordonnées. À moins de supposer que le Mirouer a été écrit seulement pour le plaisir de son auteur et d'une poignée de ses proches, il faut bien admettre qu'un tel public existait déjà en 1519 autour de Morlaix ; et qu'il s'était en 1575 élargi au point qu'une édition imprimée en était économiquement viable.

À la différence des rhétoriqueurs qui vivent en intellectuels domestiques auprès des grands seigneurs et partagent généralement le sentiment que la vie est un spectacle vide de sens que seuls peuvent encore épicer les artifices de la forme - ce que dénoncera Du Bellay ${ }^{23}$ et plus tard encore Molière -, Maître Jehan conserve la conviction qu'au-dessus de ce monde imparfait plane un ciel de cristal, de pierres précieuses et d'or auquel nous pouvons espérer accéder un jour. Et il invente cette façon originale de les mettre en regard, disposition qui a dû rencontrer un tel succès qu'on s'en souvenait encore, cinquante-six ans plus tard, à Cuburien.

On est là très loin de l'image des "clercs semi-lettrés » chère à notre regretté Christian-J. Guyonvarc'h. Et l'énigme de la non-traduction proposée en 1914 par Émile Ernault s'éclaire enfin : sous-estimant par préjugé l'auteur de ces vers, il a cru que le chaos était involontaire, symptôme d'inculture, d'incohérence de la pensée et de l'expression ; il n'a donc pas pris la peine de "remettre les choses à leur place », se privant ainsi d'un appréciable petit bonheur.

23. Joachim Du BELLAY, Défense et illustration de la langue française, 1549. 